\title{
Correction: New Stiff Matter Solutions Are Really Not Stiff
}

\author{
Salah Haggag' and Joseph Hajj-Boutros ${ }^{2}$
}

Received June 11, 1990

\begin{abstract}
Four new solutions in general relativity have recently been derived as representing static spherically symmetric stiff matter, $\rho=p$. It is pointed out that the equation of state is, in fact, $\rho+p=0$. It is further shown that two of the solutions are physically reasonable, turning out to represent the vacuum, one of them with a $\Lambda$ term.
\end{abstract}

Recently, one of us (Hajj-Boutros, 1989) presented four new exact solutions to the Einstein field equations which are static and spherically symmetric. Because of a missing minus sign in the expression for the energy density $\rho$, the equation of state appeared as that for stiff matter, $\rho=p$. Correcting this mistake, we point out that the equation of state for these solutions is, in fact, $\rho+p=0$. Since this rectification affects the characteristics of the solutions derived therein, here we reexamine each of them. It turns out that two of the four solutions are physically reasonable. The first is equivalent to the exterior Schwarzschild solution. The other leads to a constant pressure throughout the fluid, and thus could represent a vacuum solution to the field equations with a $\Lambda$ term.

Using isotropic coordinates, one finds that the static spherically symmetric metric has the form

$$
d s^{2}=e^{\lambda(r)} d \sigma^{2}-e^{\nu(r)} d t^{2}
$$

where

$$
d \sigma^{2}=d r^{2}+r^{2}\left(d \theta^{2}+\sin ^{2} \theta d \phi^{2}\right)
$$

For a perfect fluid, the energy density is then given by

$$
8 \pi \rho=-e^{-\lambda}\left(\lambda^{\prime \prime}+\frac{\lambda^{\prime 2}}{4}+\frac{2 \lambda^{\prime}}{r}\right)
$$

${ }^{1}$ Science \& Mathematics Center, P.O. Box 1070, Ta'if, Saudi Arabia.

${ }^{2}$ Physics Department, Lebanese University, P.O. Box 72, Mansourieh-El-Maten, Lebanon. 
The minus sign was missing in equation (2.5), and consequently in equation (2.10), of Hajj-Boutros (1989). It is now clear that the solutions presented there correspond to the equation of state $\rho+p=0$, and not stiff matter $\rho=p$.

Four solutions are given by

$$
e^{\lambda}=\left\{\begin{array}{l}
r^{-2} \operatorname{ch}^{2 / \alpha}(\alpha \log r+\beta) \\
r^{-2} \operatorname{sh}^{2 / \alpha}(\alpha \log r+\beta)
\end{array} \quad \alpha=\frac{1}{2} \text { or }-1\right.
$$

where $\beta$ is an arbitrary constant.

Upon simplification, (3) reduces to

$$
e^{\lambda}=\left[\frac{1}{2 b}\left(1 \pm \frac{b^{2}}{r^{2 \alpha}}\right)\right]^{2 / \alpha}, \quad \alpha=\frac{1}{2} \text { or }-1, \quad b=e^{-\beta}
$$

This leads to the following simplified expressions for the metric and energy density, respectively:

$$
\begin{aligned}
d s^{2} & =\left[\frac{1}{2 b}\left(1 \pm \frac{b^{2}}{r^{2 \alpha}}\right)\right]^{2 / \alpha} d \sigma^{2}-4\left(\frac{1 \mp b^{2} / r^{2 \alpha}}{1 \pm b^{2} / r^{2 \alpha}}\right)^{2} d t^{2} \\
8 \pi \rho & =-8 \pi p= \pm(1-2 \alpha)\left[\frac{r^{\alpha}}{2 b}\left(1 \pm \frac{b^{2}}{r^{2 \alpha}}\right)\right]^{-2(\alpha+1) / \alpha}
\end{aligned}
$$

We now consider the two cases corresponding to the two values of $\alpha$.

1. $\alpha=1 / 2$. From (6) we find $\rho=p=0$, i.e., the two solutions represent the vacuum. It is then interesting to check whether any of them represents flat spacetime or the Schwarzschild exterior solution. Equation (5) reduces to

$$
d s^{2}=\left(\frac{1}{2 b}\right)^{4}\left(1 \pm \frac{b^{2}}{r}\right)^{4} d \sigma^{2}-4\left(\frac{1 \mp b^{2} / r}{1 \pm b^{2} / r}\right)^{2} d t^{2}
$$

Applying the transformation $t \rightarrow t / 2, r \rightarrow 4 b^{2} r$, the metric (7) takes the form

$$
d s^{2}=\left(1 \pm \frac{1}{4 r}\right)^{4} d \sigma^{2}-\left(\frac{1 \mp 1 /(4 r)}{1 \pm 1 /(4 r)}\right)^{2} d t^{2}
$$

The solutions (7) are thus transformed into the Schwarzschild exterior metric in isotropic coordinates with mass parameter $m= \pm 1 / 2$, respectively (see, e.g., Kramer et al., 1980). The solution with lower sign is obviously unphysical, since it represents the gravitational field due to a negative mass.

2. $\alpha=-1$. In this case (5) and (6) reduce, respectively, to

$$
\begin{aligned}
& d s^{2}=\frac{4 b^{2}}{\left(1 \pm b^{2} r^{2}\right)^{2}} d \sigma^{2}-4\left(\frac{1 \mp b^{2} r^{2}}{1 \pm b^{2} r^{2}}\right)^{2} d t^{2} \\
& 8 \pi \rho=-8 \pi p= \pm 3
\end{aligned}
$$


Hence, the solutions represent a perfect fluid with constant pressure and density. Note that this result could have been anticipated from the equation of motion $T_{; b}^{a b}=0$, which reduces, in the metric (1), to the equation

$$
p^{\prime}+(\rho+p) \nu^{\prime}=0
$$

It is obvious that $\rho+p=0$ implies $p=-\rho=$ const. Again the solution with lower sign is unphysical because of the negative energy density appearing in $(10)$.

On the other hand, a distribution with constant pressure is only conceivable for $p=0$, namely dust and vacuum. Accordingly, for the solution with upper sign in (9) and (10) we substitute $p-3 / 8 \pi$ for $p$ and $\rho+3 / 8 \pi$ for $\rho$ into the energy tensor. It turns out that the solution could represent a vacuum solution to the Einstein field equations with a $\Lambda$ term, where $\Lambda=3 / 8 \pi$. Note that the above modification cannot apply to the solution with lower sign, since it will lead to a negative value for $\Lambda$.

In conclusion, the two physically reasonable solutions turn out to be vacuum solutions, one of them with a $\Lambda$ term. The problem for a static spherically symmetric stiff matter is yet unsolved.

\section{REFERENCES}

Hajj-Boutros, J. (1989). International Journal of Theoretical Physics, 28(1), 105.

Kramer, D., et al. (1980). Exact Solutions of Einstein's Field Equations, Cambridge University Press, Cambridge, p. 158. 